\title{
Estimation of Walking rate in Complex activity recognition
}

\author{
Hooman Kashanian \\ Department Of Computer \\ Engineering \\ Islamic Azad University \\ Ferdows, Iran
}

\author{
Saeed Sharif \\ Department Of Computer \\ Science \\ Islamic Azad University \\ Ferdows, Iran
}

\author{
Ralf Akildyz \\ Department Of Electronic And \\ Computer Science \\ Hacettepe University \\ Ankara, Turkey
}

\begin{abstract}
Physical activity recognition using embedded sensors has enabled by many context-aware applications in different areas. In sequential acceleration data there is a natural dependence between observations of movement or behavior, a fact that has been largely ignored in most analyses. In this paper, investigate the role that smart devices, including smartphones, can play in identifying activities of daily living. Monitoring and precisely quantifying users' physical activity with inertial measurement unit-based devices, for instance, has also proven to be important in health management of patients affected by chronic diseases, e.g. We show that their combination only improves the overall recognition performance when their individual performances are not very high, so that there is room for performance improvement. We show that the system can be used accurately to monitor both feet movement and use this result in many applications such as any playing. Time and frequency domain features of the signal were used to discriminate between activities, it demonstrates accuracy of $93 \%$ when employing a random forest analytical approach.
\end{abstract}

Keywords: Complex activity recognition; Mobile and ubiquitous environment; Accelerometer; Cell Phones; Humans; Monitoring; Ambulatory, random forest, Online prediction. 


\section{INTRODUCTION}

STEOARTHRITIS (OA) is a degenerative disease causing pain, Ojoint stiffness, loss of function and disability [1]. The knee is one of the most commonly affected joints disabling a large proportion of the adult population over a range of daily activities [2].

Exercise is recognized as a key component in the management of knee OA [3] but its effectiveness in restoring joint function is hampered by a lack of individualized programs and by low treatment fidelity. Research studies have quantified the effect of different treatment options in reducing pain and disability reporting small to moderate effects over control groups [4]-[8]. However, the delivered exercise therapies were not tailored to patients' specific impairments or their aspirations, and this may be why none of the studies have reported a definite impact of exercise on quality of life and functional outcomes. Furthermore, the long-term impact of exercise on cessation of the intervention is frequently lost or significantly reduced or simply not reported [8], [9]. This indicates a paucity of longitudinal studies into the effect of optimal rehabilitative approaches and even fewer studies addressing how to optimize the short and long-term exercise compliance in this population group.

Individualized programs can be obtained based on objective measures of patients' joint functional status; however, the routine collection of these measures is rare with the output seldom accessible or made meaningful to healthcare professionals. Simple solutions to enhance compliance may be achieved by solving organizational and accessibility issues (e.g. location, time, work and other commitments) and addressing cost concerns. Furthermore, providing patients with marker of performance and ensuring a correct understanding of the content of rehabilitation will keep them motivated while supporting self-management [10], [11].

It is expected that by prescribing patients exercise regimes based on sound biomechanical assessed deficits and providing them with targets and feedback on performance will enhance compliance and hence treatment effectiveness [12], [13]. Objective measures of knee functional status, referring mainly to knee 3-D angles, are generally obtained in laboratories using expensive, time consuming and difficult to operate equipment. Moreover, the retrievable information is related to an artificial environment over a short period of time. On the other hand, the clinical benefit for long-term monitoring of patients in everyday situations has been advised and it has been proposed that it should be used to inform treatment [14], [15]. Longterm monitoring within each patient environment can only be possible with the use of an ambulatory monitoring system. However, to be effective, this technology needs to be able to inform clinicians on patients' joint status and, be simple and easy to use for patients and allow them to gain feedback on their performance. Despite the use of wearable technology and particularly inertial measurement units (IMUs) gaining popularity within the research environment, clinical uptake remains poor [16], [17].

The main advantage of using wearable devices over standard laboratory-based motion analysis systems to track joint movement relates to the portability of the instrument allowing for prolonged data collection in more realistic environments. However, their everyday use is still limited by poor patient acceptance. To obtain knee angles from IMUs two devices have to be positioned on the shank and thigh of the subject to extrapolate the relative movement between the two segments. The output accuracy is affected by drift from required integration of acceleration and angular velocity values and, artefacts errors due to skin movement and misalignments [18], [19]. In addition to this, their use still requires a certain level of expertise that can limit wide adoption especially in the ageing population. More simple activity monitors based on accelerometry are common and readily accessible on the market for a range of applications. However, the measures obtained are frequently limited to how active a patient is, and few are able to discriminate between activity performed, or able to record step counts and distance travelled. Although important for general activity levels, these parameters do not represent clinically relevant measures directly related to knee joint status. For rehabilitative purposes, it would be important to be able to monitor knee function (e.g., Knee kinematics).

Within our group we explored the use of a flexible conductive polymer material as a sensing modality for knee movement [20]. Laboratory experiments were conducted to evaluate the polymer sensor in measuring flexion and extension angles of the knee in a controlled environment where the knee movement was restricted and standardized with a dynamometer. A subject specific algorithm was defined to obtain measures of knee flexion and extension angles to an accuracy of $1^{\circ}$ with the gold standard [20]. The previous study characterized the sensor and validated it in a controlled laboratory setting, but no investigations were conducted to evaluate the sensor's response to free, unconstrained movement. With the intended use of the sensor for knee rehabilitation in the home and clinics, further testing is required to evaluate the sensor capability to follow knee movement patterns in dynamic real life conditions. It was also essential that the sensor had low power requirement to facilitate continuous data acquisition.

The aim of this study was to investigate the reliability of the response of the sensor to everyday tasks and to evaluate its potential towards assessing joint range of motion and activity identification. To support out-of-the laboratory assessments, wearable electronics were developed in the form of a sensing node to allow wireless data acquisition from the sensor. Design constraints included the need for the system to be unobtrusive, low cost, low power and simple to use. This paper focuses on the evaluation and exploitation of the system in reference to the output from a flexible polymer sensor embedded in a pair of leggings. The main contributions relate to the system ability to demonstrate activity discrimination based on a single passive polymer sensor and simultaneously derive a surrogate of knee range of motion from the sensor output to comprehensively describe knee functional status during a specific activity context. 

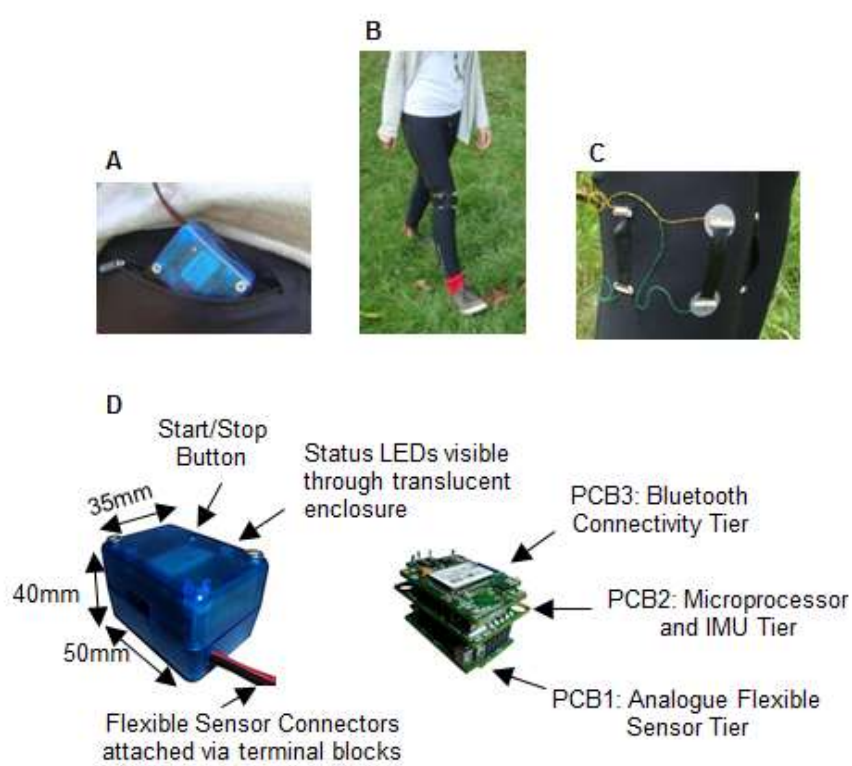

Fig.1. Photographs showing (A) wireless node positioned in the back pocket, (B) sensor integration to a pair of leggings and (C) detail of flexible sensor unit. (D) Photos showing node package with overall dimensions (left) and assembly of printed circuit boards (right).

Two exploitation cases were considered: (i) one that necessitates a subject specific calibration, based on a simplified approach to activity discrimination and (ii) an approach that eliminates the individual calibration but incorporates computational resources for a machine learning approach. Individual subject activity discrimination was successfully achieved based on an innovative combination of two spectral features, median frequency and total power of the spectrum, while group classification was achieved with high accuracy based on a random forest algorithm. Independently of the exploitation set-up of choice, by proving the capability of the system in monitoring knee function in everyday life scenarios, with an appropriate feedback interface, it will be a valuable tool to support knee rehabilitation by providing objective measure of function to clinicians as well as enhancing long term patients' compliance and promoting selfmanagement.

\section{METHOD}

\subsection{Smart Leggings}

The sensor unit (Fig.1.C) consists of a conductive flexible polymeric material in the form of a thin $(0.2 \mathrm{~mm})$ rectangular strip $(50 \mathrm{~mm} \times 100 \mathrm{~mm})$. The conductivity is provided by the presence of graphitized carbon black Nano powder particles $(<500 \mathrm{~nm})$ in a polyurethane substrate. The ratio between the two compounds in the conductive polymer composite is 20:80. Two connectors were attached at each end of the sensor unit. The sensor was secured on to a pair of commercially available leggings ( $92 \%$ polyester, $8 \%$ elastin) (Fig.1.B), in a pre-stretched condition, to coincide with the anterior aspect of the knee joint. The composite material has a resistor likefunction so when stretched, it changes resistance. Knee motion stretches the sensor allowing for a direct sensing modality for knee flexion/extension movement.

\subsection{Data Acquisition: Multi Sensors Wireless Platform}

Data from the sensor unit were acquired by means of a custom wireless sensing node (Fig.1.A, D). The developed sensor node consists of three printed circuit board (PCB) tiers (Fig.1.D), each with its own functionality as follows:

i. PCB 1: analogue interface tier accommodating circuitry for the flexible sensor unit; a Wheatstone bridge configuration is used to detect resistive changes within the flexible sensor, the signal is then further amplified by a micropower precision instrumentation amplifier (LT1789, Linear Technology, Milpitas, CA, USA) before being converted to digital values;

ii. PCB 2: core tier with a microprocessor (64MHz PIC18F family, Microchip Technology Inc., Chandler, AZ, USA) and an inertial measurement unit (IMU) embedding a 3 axis accelerometer (ADXL345, Analog Devices Inc, Norwood, MA, USA) and 3 axis gyroscope (L3G4200D, STMicroelectronics, Geneva, Switzerland);

iii. PCB 3: connectivity tier incorporating a small form factor, low power Bluetooth module (RN42, Microchip Technology Inc., Chandler, AZ, USA) allowing wireless data transmission for distances up to 20 meters. Data were acquired synchronously from the IMU and flexible sensor unit at $122 \mathrm{~Hz}$ sampling frequency;

The PCB tiers are encased in a box with sides of $35 \mathrm{~mm} \times 50 \mathrm{~mm} x$ $40 \mathrm{~mm}$ (width $\mathrm{x}$ length $\mathrm{x}$ height). The node operates on a $3 \mathrm{~V}$ battery and its overall mass is $54 \mathrm{~g}$. During testing the node was placed on the back pocket of the leggings. Thin wires sewn along the seam of the leggings connected the sensor unit to the PCB 1 of the wireless node.

\subsection{Participants}

Twelve healthy subjects with no reported knee pain (Age: $27 \pm 5$ years, Height: $1.7 \pm 0.1 \mathrm{~m}$, Body Mass: $66 \pm 12 \mathrm{~kg}$ ) took part in the study. The sample size was defined in accordance with earlier recomendations [21]. For a power of $80 \%$ and to achieve a specificity of $95 \%$, and assuming test-retest reliability of 0.9 for the sensor outputs with two observations, a sample size of 12 would suffice to allow for observations of test-retest reliability of 0.6 or greater. Written informed consent was obtained from all subjects prior testing, following attainment of ethical approval. Ethical approval was granted by the Imperial College Ethics Research Committee.

\subsection{Experimental Procedures}

Each participant was tested on two separate occasions with at least a one-week gap between sessions, referred in the text as Test 1 and Test 2. A test session consisted of the participant walking and running both indoors and outdoors, and going up and down consecutive flights of stairs. The indoor test took place along a $30 \mathrm{~m}$ corridor; and participants were asked to walk and run this distance 10 times. The stair test was conducted in a public building back stair case using 5 consecutive flights of stair with 10 steps each (width $30 \mathrm{~cm}$, height $16 \mathrm{~cm}$ ) with subjects being requested to go up and down the stairs two times. This allowed 10 data sets for both ascending and descending the stairs. The outdoors test was conducted in a quiet nearby park and subjects were instructed to walk and run without stopping for two minutes, twice, with sufficient rest periods allowed between tests. Each subject performed the different activities at their preferred, comfortable speed. During each session, participants were asked to wear the smart leggings and to position the sensor unit to 
cover the anterior aspect of their right knee. This imitates the use of the system in home environments where users are unsupervised allowing to evaluate the system in real condition. The sensing node was positioned by the investigator in the back pocket of the leggings once Bluetooth connection was established with a notebook (HP Mini 5103 Notebook PC, Hewlett-Packard Company, Palo Alto, CA, USA) for data acquisition. A test session lasted approximately 45 minutes.

\subsection{Data Pre-Processing and Sensor Output}

The wearable system allows for simultaneous multisensor data collection, but for the aim of the current study only the flexible sensor unit output was analyses. The use of accelerometer and gyroscopes data is already well established and widely accepted for activity monitoring whereas, the novelty of the present study resides in the ability to provide direct information of knee function while characterizing activities performed using a single passive polymer sensor. Data were pre-processed by filtering and having the DC offset removed from the signal output. A 4th order Butterworth filter with $10 \mathrm{~Hz}$ cut-off frequency was used. Time histories of the signal outputs were analysed to investigate the capability of the sensor to monitor dynamic knee movement. The range of the signal output in the time domain was evaluated. This range can be considered as a surrogate of the knee range of motion, since the sensor stretches as the knee bends, generating the output which allows mapping the knee flexion/extension movement. Range was normalized to each subject's leg length [22]. Test-retest reliability of the signal output range was assessed by mean of intra-class correlation as defined by Shrout and Fleiss [23] and examined accordingly to the classification of Landis and Kock [24]. Bland and Altman tests statistics [25] were performed to provide a measure of agreement between tests. All data processing and statistical analysis were completed with Matlab (The MathWorks Inc., Natick, MA, USA) and SPSS (SPSS Inc., Chicago, IL, USA) software.

\subsection{Spectral Domain Activity Discrimination}

A frequency domain approach was adopted to discriminate between activities. A single-sided power spectral density (PSD) analysis was performed using the periodogram method over the whole signal recorded per trial. From the PSD function $(S(f))$, total power of the spectrum and median frequency (MDF) were computed. The total power of the spectrum $(\mathbb{P})$ is the cumulative power of the signal:

$$
P=\int_{0}^{\infty} s(f) d f
$$

The median frequency is the frequency dividing the signal power spectrum into two equal halves:

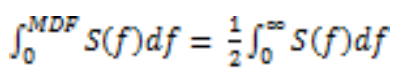

These two parameters were used as discriminative features to classify tasks performed. This approach was taken to verify if a simple discriminative algorithm using only these two parameters would allow activity differentiation instead of using computationally complex algorithms involving machine learning techniques.

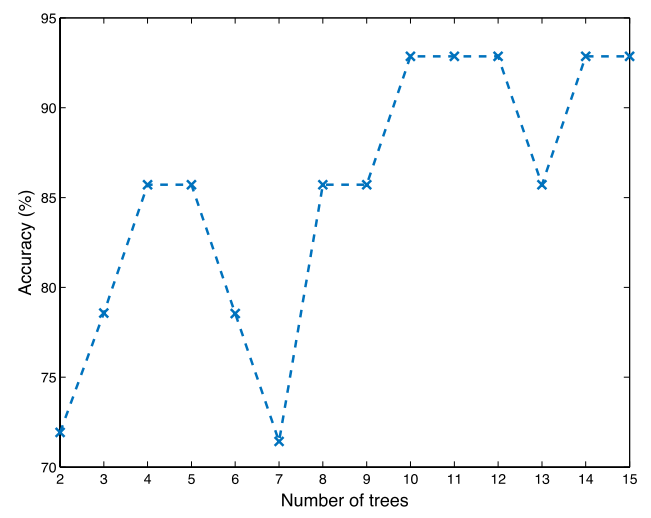

Fig.2. Graph showing the relationship between accuracy and number of trees. descent (upper right), running (lower left) and, walking (lower right).

Table 1

TEST-RETEST RELIABILITY AND BLAND AND ALTMAN TEST RESULTS

\begin{tabular}{|c|c|c|c|c|c|c|c|}
\hline & \multicolumn{2}{|c|}{ Test-Retest Reliability } & \multicolumn{5}{|c|}{ Bland and Altman Test } \\
\hline & ICC Coefficient & $\begin{array}{c}95 \% \mathrm{Cl} \\
\text { (Lower,Upper } \\
\text { Bound) }\end{array}$ & $\begin{array}{c}\text { d: } \\
(\mathrm{mV} / \mathrm{m})\end{array}$ & $S D_{\bar{I}}$ & $\begin{array}{c}S E_{\bar{x}} \\
(\mathrm{mV} / \mathrm{m})\end{array}$ & $\begin{array}{c}\text { Repeatability } \\
\text { Coefficient } \\
(\mathrm{mV} / \mathrm{m})\end{array}$ & $\begin{array}{c}\text { 95\% LOA } \\
\text { (Lower,Upper } \\
\text { Bound) }\end{array}$ \\
\hline Run Indoors & 0.958 & $0.860 ; 0.988$ & 11.0 & 11.2 & 3.2 & 21.9 & $-10.9 ; 32.9$ \\
\hline Run Outdoors & 0.984 & $0.945 ; 0.995$ & 1.0 & 11.8 & 3.4 & 23.1 & $-22.1 ; 24.1$ \\
\hline Walk Indoors & 0.897 & $0.657 ; 0.970$ & -0.5 & 8.9 & 2.6 & 17.5 & $-18.0 ; 17.0$ \\
\hline $\begin{array}{c}\text { Walk } \\
\text { Outdoors }\end{array}$ & 0.958 & $0.861 ; 0.988$ & -0.9 & 4.2 & 1.2 & 8.3 & $-9.2 ; 7.4$ \\
\hline $\begin{array}{l}\text { Stair Ascent } \\
\text { at.com }\end{array}$ & 0.867 & $0.557 ; 0.961$ & -3.6 & 14.4 & 4.2 & 28.2 & $\begin{array}{r}-31.9 ; 24.6 \\
571\end{array}$ \\
\hline Stair Descent & 0.938 & $0.796 ; 0.982$ & -0.6 & 9.3 & 2.7 & 18.1 & $-18.8 ; 17.5$ \\
\hline
\end{tabular}



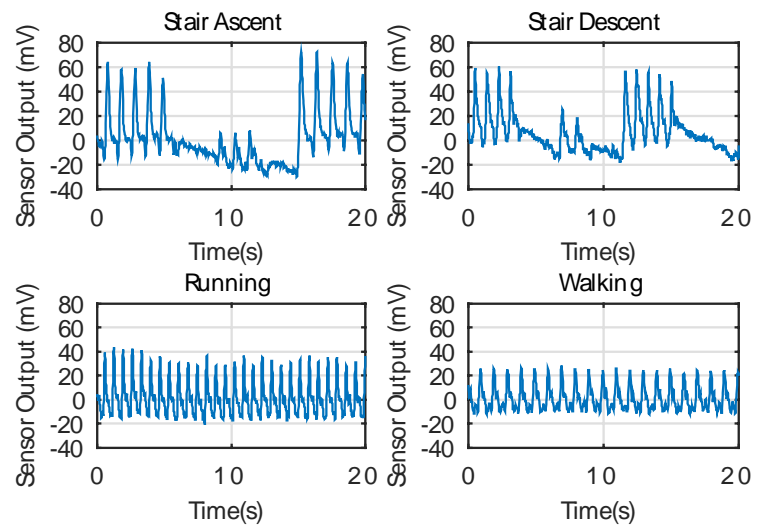

Fig.3. Sensor output time histories during stair ascent (upper left), stair

The sensor capability in discriminating activity was analysed treating participants individually as well as a group for the two tests conducted. This was done to investigate if a general activity detection algorithm could have been defined based on a simple classification method that would not require subject specific calibration (e.g.: identify specific thresholds for each subject to allow real time classification). Median frequency and total power of the spectrum were normalized to each individual's anthropometric features (leg length, body mass and height) when comparing data across participants using the method proposed by Hof [22]. Data analysis was performed using Matlab software. The data showed the need for subject specific calibration when only MDF and power of the spectrum were used for activity classification as no general-purpose thresholds could be defined that would have satisfied all participants' data. Machine learning was then utilized to tackle this problem and overcome the necessity of a baseline subject calibration.

\subsection{Random Forest Activity Classification \\ A Random Forest [26] was used to develop a generalized}

classification method to discriminate between activities based on features extracted from the flexible sensor output. Random forests are statistical modern machine learning techniques that allow accurate classification of large datasets that are screened by independent trees, in this instance, classification trees, which form the forest. Each tree develops upon a set of rules based on discriminatory features randomly selected from measured parameters. Random forests perform feature selection automatically to develop each tree that can alternatively be expressed as set of rules. In each node of a tree, a decision is made based on one feature. The random forest combines the response of each tree via majority voting to obtain the ultimate classification response.

The random forest employed in this study is an ensemble of 10 classification decision trees. The number 10 was decided by verifying that increasing the number of trees did not affect

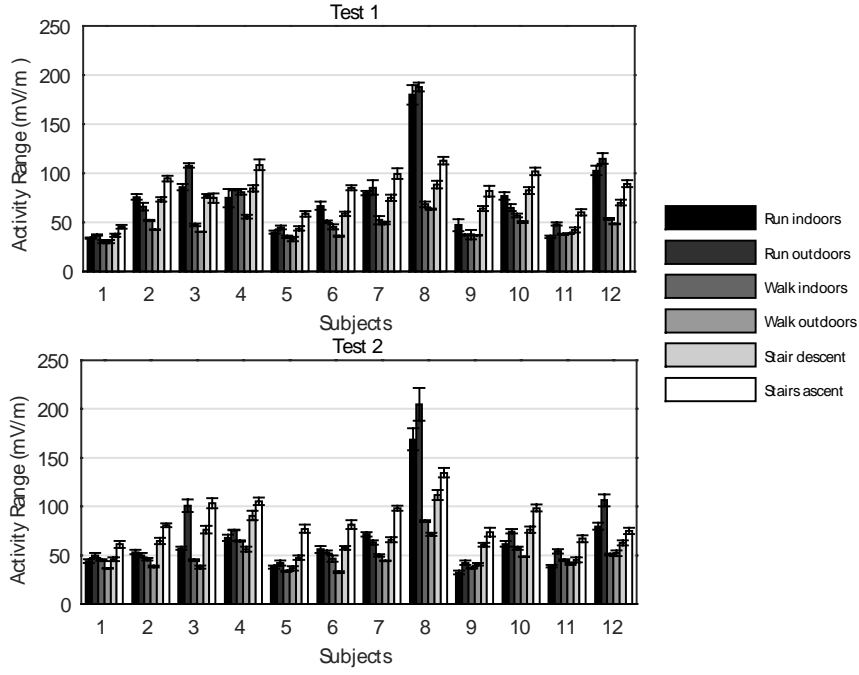

Fig.4. Sensor output range as surrogate of knee range of motion during the different activities for both tests conducted.

accuracy significantly (Fig.2) but, increased the computational complexity of the method. Our aim is to keep the complexity of the data processing to a minimum to allow a timely real time data visualisation in the future.

The ensemble classifies activities into walking, running, and ascending and descending stairs. The ensemble was provided with features from time and frequency domain analysis of the sensor output namely: MDF, power of the spectrum, peak frequency, maximum spectral amplitude and output range of the signal in the time domain. Anthropometric parameters, gender, age, height and leg length, were also utilised. $90 \%$ of the data were randomly selected and used for the construction of the trees and $10 \%$ of the data were used to test the algorithm.

Performance metrics consisting of accuracy, specificity, sensitivity, and $\mathrm{F}$ measure were computed from the confusion matrix to evaluate the classification method. This analysis was performed using Matlab Statistics Toolbox.

\section{RESULTS}

Typical time series of the sensor output are plotted in Fig.3 for the different activities performed, $20 \mathrm{~s}$ time frames are depicted. These plots show that the sensor is able to follow the knee movement during dynamic tasks by capturing the knee flexion/extension repetitions throughout the trials. The output, presented in $\mathrm{mV}$, can thereby be considered a surrogate of knee sagittal kinematics.

The range of the measured voltage from the sensor is shown in the bar charts in Fig. 4 for both tests conducted for each subject. No statistical significant differences were found within subject $(p>$ 0.05 ). This range could be considered a surrogate of knee range of motion as it quantifies the amount the sensor has stretched due to knee movement during each performed task.

An almost perfect test-retest reliability (ICC > 0.8) was obtained for the output range among all participants (Table I). Bland and Altman test results in Table I and Fig.5 demonstrate good to high agreement between tests with the majority of 
International Journal of Computer Applications Technology and Research

Volume 5- Issue 9, 561 - 569, 2016, ISSN:- 2319-8656
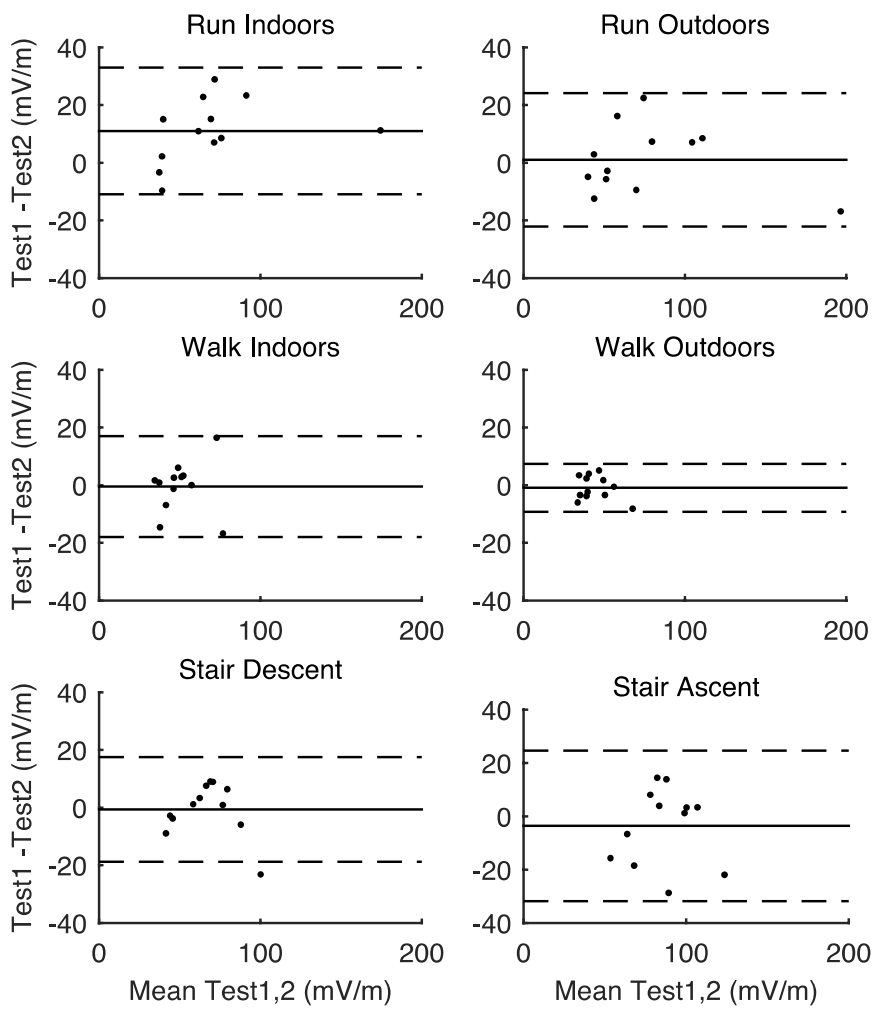

Fig.5. Bland and Altman plot of agreement between Test 1 and 2. Dashed lines represent upper and lower limit of agreement and the solid line represents the mean difference.

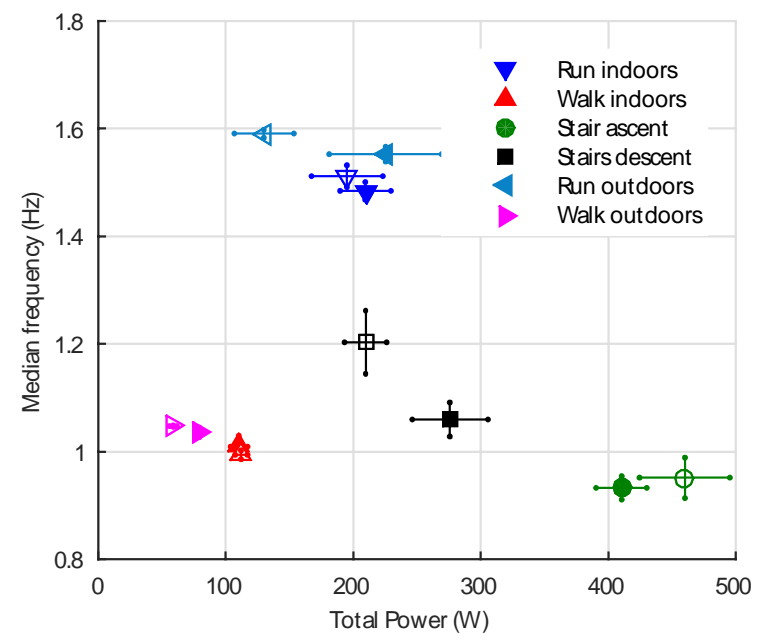

Fig.6. Activities discrimination using MDF and Total Power of the spectrum. Filled markers refers to Test 1, unfilled ones to Test 2 for one participant. The bars indicate \pm one standard deviation. data points falling within the locus of agreement. The highest biased $\left(\mathrm{d}^{-}=11 \mathrm{mV} / \mathrm{m}\right)$ was observed for running indoors indicating higher variations occurred in this task. This could be related to the intrinsic variability of the movement but also to the fact that the sensor may have been prone to major movement artefacts with respect to the underlying knee during this fast task which was repeated 10 times.

Fig.6 shows an example of activity clustering for one participant when only using MDF and the total power of the spectrum as discriminative features. Comparable results are obtained for Test 1 and 2. Similar clustering was observed for other participants; summary values of normalized MDF and total power of the spectrum (with standard deviation indicated in brackets below each value) are shown in Table 2.

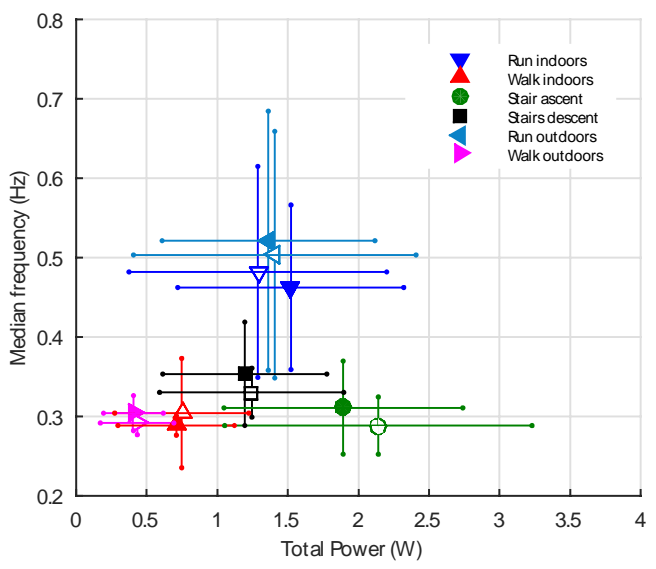

Fig.7. Activities discrimination using MDF and Total Power of the spectrum. Filled markers refers to Test 1 , unfilled ones to Test 2 for all participants. The bars indicate \pm one standard deviation. Data are normalised to subject specific anthropometric parameters.

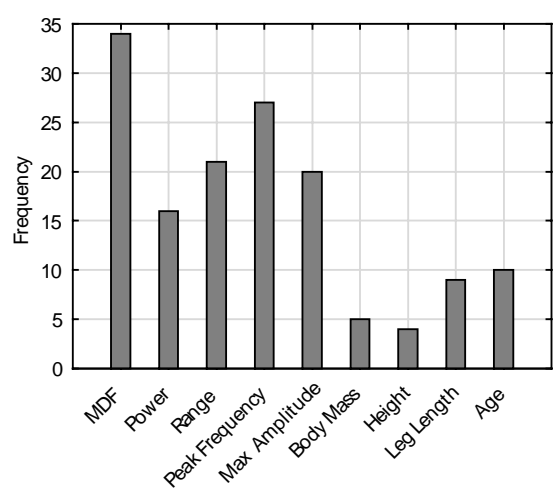

Fig.8. Histogram showing the frequency of the features selected by the Random forest for its decision trees.

Considering the participants as a group led to the discriminatory ability of the MDF and power of the spectrum to be lost (Fig. 7). This 
occurred despite normalization of the outputs to subject specific anthropometric parameters [22].

Subject specific calibration is therefore required if these two parameters are to be used alone to identify activities and define thresholds boundaries for the different activities performed.

The possibility of using a machine learning approach, in particular a random forest algorithm, was investigated to allow a generalized discrimination between activities from the sensor output, avoiding the need for subject specific calibration. An ensemble of 10 trees was created using 9 features (Table 2).

Table 2

\section{RANDOM FOREST PERFOMANCE METRICS AND FEATURES}

\begin{tabular}{lllll}
\hline \hline Accuracy & \multicolumn{2}{l}{ S2.8\% } & \\
\hline & Run & Walk & $\begin{array}{l}\text { Stair } \\
\text { Descent }\end{array}$ & $\begin{array}{l}\text { Stair } \\
\text { Ascent }\end{array}$ \\
\hline Sensitivity & 1 & 1 & 0.75 & NaN \\
Specificity & 1 & 1 & 1 & 0.92 \\
F-score & 1 & 1 & 0.85 & 0 \\
\hline \# of & 9 & & \\
features & & & \\
\hline Features & $\begin{array}{l}\text { Median Frequency, Total Power of the } \\
\text { Spectrum, Peak Frequency, Max Amplitude, } \\
\text { Output Range, Body Mass, Height, Leg } \\
\text { Length, Age }\end{array}$ \\
\hline \hline
\end{tabular}

Moreover, data from the two tests were combined to allow for more data during the training phase of the random forest. The ensemble, when tested with the remaining $10 \%$ of the sample data, performed well with an accuracy of $93 \%$. This implies $93 \%$ of the time activities were correctly identified. Detailed performance metrics of the ensemble are shown in Table III.

Fig.8 shows the frequency of occurrence of features automatically selected by the decision trees (i.e.: how many times each feature is picked across all nodes for all trees). Among these features MDF is the most important one, whereas anthropometric parameters, and particularly body mass and height, do not play an important role in the classification process.

\section{DISCUSSION}

A novel wearable system has been presented that allows simultaneous estimation of a surrogate for knee range of motion and identification of activity type performed. The sensor unit was able to reliably detect knee movement during dynamic activities at different speeds as shown in Table I. The excellent reliability demonstrated the sensor is not affected by movement artefacts allowing for valid results despite don-doff of the system and participants positioning it. This offers the potential of the system to support rehabilitation however, without further work to understand the relationship of the surrogate to knee range of motion it may have limited utility as an outcome measure at this stage.

The time series waveforms of the sensor output (Fig.3) recall typical knee kinematics curves reported in the literature [27], [28]. This indicates the potential to use the sensor output as a surrogate for knee sagittal kinematics, as the output is the direct response to stretching caused by knee flexion/extension movements, thereby permitting acquisition of data from unconstrained environments over extended periods of time. It follows that the range of motion required to perform activities of daily living can also be inferred from the sensor output. Repetitive patterns (Fig.4) were observed among participants in the sensor output range reflecting the knee joint angulation expected for the activities performed [27], [28], lower values indicate the sensor has been stretched less responding to the demand of the activity in requiring less knee flexion/extension. The findings show that walking was the activity that required the smallest range of movement (smaller stretching of the sensor) whereas stair ascent the one with the greatest range of knee motion (greater stretching of the sensor) in agreement with range patterns reported in biomechanical studies [27], [28]. For three of the participants tested, however, running showed the highest stretching span as can be observed from Fig.4, this may be due to the fact that these participants were recreational sport runners and this may be associated to a greater knee flexion/extension range of motion [29]. The knee range of motion is expressed in $\mathrm{mV}$ for this preliminary investigation as a first step to identify the capability of the sensor to track knee movement dynamically; the next step will be to identify the relation between the sensor output $(\mathrm{mV})$ and knee angles $\left(^{\circ}\right)$ captured through a 3-D motion analysis system to allow the representation of the output in degrees. However, the possibility to use the output in $\mathrm{mV}$ as representation of knee sagittal angles will be explored further together with the clinical interpretation. A database of healthy knees movement, monitored in $\mathrm{mV}$, can be acquired to allow for comparison with pathological knees in the future or, similarly, if we have a baseline measure of a patient knee angles in $\mathrm{mV}$ based on the sensor data and having proved, in this study, the accuracy and repeatability of the sensor outputs, the sensor can be used to monitor knee function over time as relative comparison to each individual baseline measure. This also aligns with the idea that functional improvements are relative and specific to each subject. Therefore, there exists a situated use for the sensor in monitoring knee movement also if expressed in $\mathrm{mV}$.

Knee range of motion is often evaluated during the clinical assessment of patients with knee OA with the use of goniometers via a static end range of motion passive test and has frequently been reported as clinically significant parameter in studies of the knee OA population [30]. However, such data and the majority of research data are one off measurements performed within a laboratory or clinical environment and as such not representative of everyday tasks in real life settings. In this study, differences in participants' performances could be appreciated between a task performed indoor or outdoor. An improved understanding of knee function, in real life contexts would permit more effective evaluation of a patient's functional limitations 
that could be used to prescribe targeted exercise regime to improve specific functions and follow-up patients' progresses. This may be facilitated by the described system. The system allows continuous long-term monitoring of the knee, which can be expressed as surrogate of knee range of motion, and furthermore it allows the context of the activity to be identified accurately.

Firstly, a simple classification method using MDF and total power of the spectrum was investigated for the identification of activity. This proposed method prioritized the simplified approach (based on only two features) despite the need for subject specific calibration. Different aspects of a signal, and generally of an acceleration signal both in time and frequency domains have been explored to detect activities [31], [32], some of which requiring computationally intense algorithms. MDF discriminatory ability was proposed before for the analysis of acceleration data [33]. MDF alone would not suffice for discrimination between activities using the proposed sensor output; thereby, in this study, it was used in conjunction to the total power of the spectrum. These two features were chosen as they incorporate significant discrimination capability. Good activity discrimination was achieved: data formed defined classes accordingly to the activity performed (Fig.5). This was particularly evident when the participants were analyzed separately, on a subject-by-subject basis. Net separation between activity classes was not achieved when data for all subjects were treated together, as a group, indicating the need for subject specific calibration (Fig. 7). Although sensors could be calibrated for each subject, this may represent a limitation for future clinical adoption, as an additional step is required before actual use, implying extra economic and time costs. This was resolved by successfully employing a random forest algorithm to automatically detect activities in a generalised fashion. This method was mathematically more complex but has the advantage that can be applied without the need for subject specific calibrations.

Machine learning techniques, among which random forests, have been recently used to classify activities from acceleration data acquired via a number of 3-axis accelerometers or smartphone positioned on different parts of the body [31],[32], [34]-[39]. Most of the studies involved the simultaneous use of two or more devices in different positions to increase the accuracy of the classification methods proposed. This leads to a bulkiness of the system not compatible with patients' preferences [40]. On the other hand, the use of one sensor alone implied specific positioning on areas that could interfere with activities of daily living (e.g.: chest, bulky phone in the pocket) or more visible to the other (e.g: ear) against patients' discreetness. The classification method proposed showed high accuracy (93\%) utilising 9 features from a single sensor alone while allowing discrete data monitoring. The accuracy achieved compares well with the accuracy reported in previously conducted studies (range $80-99 \%$ ) using more conventional acceleration signals to detect activity. Further improvements in the accuracy may be achieved via investigating a larger set of subjects that covers the pathological case as well.

The feature that played the greatest role in the activity classification was the MDF. All the features utilised allow for an easy implementation. The random forest demonstrated good discrimination ability in correctly identifying activities performed as seen in the performance metrics table (Table III). Among the testing set values none of the data referred to stair ascent thereby explaining the low F score and sensitivity values. A larger data set will be collected to further test the method proposed having demonstrated the viability of the system for activity classification through this study.
Also, once the random forest is trained, the identification of activities for future subjects can be achieved in real time. The features utilized will be calculated to allow real-time feedback in an automated fashion by using a moving window method as the data are collected and, not over whole trial as conducted for this study. Visual feedback of the data for patients and clinicians will complement the wireless system to allow an easy and fast interpretation of the data for clinical use. Data will be made available via smartphone/tablet application or in the form of a one-page report on patient progress. Although accelerometers are established systems for activity recognition or activity level quantification in their simplest form, the sensor proposed allows also for range of movement estimation not achievable with one accelerometer. This dual functionality represents an advantage of our system over existing technology. Although the smart leggings utilised for this study still shows visible electronics, these will be integrated into clothing in the next prototype to comply with patients' needs and maximise acceptance [40]. Moreover, the system proposed requires minimum training for the end user to permit independent utilization.

\section{CONCLUSION}

Findings from this study demonstrate the feasibility of the novel sensing system in monitoring knee movement and classifying activities of daily living. Being able to monitor knee functional status outside laboratory environments will bring great advantage to the rehabilitation of patients with knee OA. Objective measures of knee health can both inform treatment and motivate patients to comply with prescribed rehabilitation regimes to enhance clinical benefit.

Additional activities will be included in further testing to have a more comprehensive classification of activity of daily living and to explore the possibility to express the output in degrees. System design together with a visual feedback tool will be improved to reflect end users preferences, both patients and health professionals, and ultimately progress into clinical adoption. The use of the sensor can also be expanded to the monitoring of clinically used performance tests to assess patients' physical function. A study conducted within our group showed the ability of the sensor to monitor performance during exercises extrapolated from a knee OA rehabilitation class [41]. Assessment of performance-based tests as suggested by OA guidelines could be included as additional processed outcome of the sensor increasing the clinical usefulness of the information obtained from the novel system.

\section{REFERENCES}

[1] J. J. Guiry, P. van de Ven, and J. Nelson, "Multi-Sensor Fusion for Enhanced Contextual Awareness of Everyday Activities with Ubiquitous Devices," Sensors, vol. 14, no. 3, p. 5687, 2014.

[2] C. Giannella, J. Han, J. Pei, X. Yan, and P. S. Yu, Mining Frequent Patterns in Data Streams at Multiple Time Granularities. MIT Press, 2003, ch. 3.

[3] G. Doretto, A. Chiuso, Y. N. Wu, and S. Soatto, "Dynamic Textures," Int. J. Comput. Vis., vol. 51, no. 2, pp. 91-109, 2003.

[4] Y. Hanai, J. Nishimura, and T. Kuroda, "Haar-like filtering for human activity recognition using $3 \mathrm{~d}$ accelerometer," in Proc. IEEE 13th Digit. Signal Process. Workshop, 5th IEEE Signal Process. Educ. Workshop, Jan. 2009, pp. 675-678 
[5] Guiry, J.J.; van de Ven, P.; Nelson, J. Multi-Sensor Fusion for Enhanced Contextual Awareness of Everyday Activities with Ubiquitous Devices. Sensors 2014, 14, 5687-5701.

[6] Tzu-Yi Hung, Jiwen Lu and Yap-Peng Tan, "Graph-based sparse coding and embedding for activity-based human identification," 2013 IEEE International Conference on Multimedia and Expo (ICME), San Jose, CA, 2013, pp. 1-6.

[7] M. Yang, L. Zhang, X. Feng, and D. Zhang, "Fisher Discrimination Dictionary Learning for Sparse Representation," in Proceedings of the 2011 International Conference on Computer Vision, 2011, pp. 543-550.

[8] L. Liu, C. Shen, L. Wang, A. van den Hengel, and C. Wang, "Encoding High Dimensional Local Features by Sparse Coding Based Fisher Vectors," Nov. 2014.

[9] R. Raina, A. Battle, H. Lee, B. Packer, A. Y. Ng, Self-taught learning: transfer learning from unlabeled data, in: Proc. Int. Conf. on Machine Learning (ICML), 2007.

[10] S. Bhattacharya, P. Nurmi, N. Hammerla, and T. Plötz, "Using unlabeled data in a sparse-coding framework for human activity recognition," Pervasive Mob. Comput., vol. 15, pp. 242-262, 2014.

[11] V. Kumar, C. Narasimham, and B. Sujith, "Classification of Time Series Data by One Class Classifier using DTW-D," Procedia Compute. Sci., vol. 54, pp. 343-352, 2015.

[12] R. J. Martin, "A metric for ARMA processes," IEEE Trans. Signal Process., vol. 48, no. 4, pp. 1164-1170, Apr. 2000.

[13] Z. Dong, W. Liang, Y. Wu, M. Pei, and Y. Jia, "Nonnegative correlation coding for image classification," Sci. China Inf. Sci., vol. 59, no. 1, pp. 1-14, 2016.

[14] A. Ravichandran, R. Chaudhry, and R. Vidal, "Categorizing Dynamic Textures Using a Bag of Dynamical Systems," IEEE Trans. Pattern Anal. Mach. Intel., vol. 35, no. 2, pp. 342-353, Feb. 2013.

[15] M. Shoaib, S. Bosch, O. D. Incel, H. Scholten, and P. J. M. Havinga, "Fusion of smartphone motion sensors for physical activity recognition.," Sensors (Basel)., vol. 14, no. 6, pp. 10146-76, Jan. 2014.

[16] M. Shoaib, S. Bosch, H. Scholten, P. J. M. Havinga and O. D. Incel, "Towards detection of bad habits by fusing smartphone and smartwatch sensors," Pervasive Computing and Communication Workshops (PerCom Workshops), 2015 IEEE International Conference on, St. Louis, MO, 2015, pp. 591-596

[17] M. Shoaib, S. Bosch, O. D. Incel, H. Scholten, and P. J. M. Havinga, "Complex Human Activity Recognition Using Smartphone and Wrist-Worn Motion Sensors.," Sensors (Basel)., vol. 16, no. 4, p. 426, Jan. 2016.

[18] S. G. Trost, Y. Zheng, and W.-K. Wong, "Machine learning for activity recognition: hip versus wrist data," Physiol. Meas., vol. 35 , no. 11, p. 2183, 2014

[19] M. Stikic, D. Larlus, and B. Schiele, "Multi-graph Based Semisupervised Learning for Activity Recognition," in 2009 International Symposium on Wearable Computers, 2009, pp. 85-92.

[20] M. Stikic and B. Schiele, "Location and Context Awareness: 4th International Symposium, LoCA 2009 Tokyo, Japan, May 7-8, 2009 Proceedings,” T. Choudhury, A. Quigley, T. Strang, and K. Suginuma, Eds. Berlin, Heidelberg: Springer Berlin Heidelberg, 2009, pp. 156-173.

[21] H. Liu, L. Yu, W. Wang, and F. Sun, "Extreme learning machine for time sequence classification," Neuro computing, vol. 174, Part A, pp. 322-330, 2016.

[22] J. R Munkers, Topology vol. 2: Prentice Hall Upper Saddle River, 2000
[23] L. R. Rabiner, "A tutorial on hidden Markov models and selected applications in speech recognition," Proc. IEEE, vol. 77, no. 2, pp. 257-286, Feb. 1989.

[24] H. Ney, D. Mergel, A. Noll and A. Paeseler, "A data-driven organization of the dynamic programming beam search for continuous speech recognition," Acoustics, Speech, and Signal Processing, IEEE International Conference on ICASSP '87. 1987, pp. 833-836.

[25] M. Yang, L. Zhang, X. Feng, and D. Zhang, "Fisher Discrimination Dictionary Learning for Sparse Representation," in Proceedings of the 2011 International Conference on Computer Vision, 2011, pp. 543-550.

[26] L. Liu, C. Shen, L. Wang, A. van den Hengel, and C. Wang, "Encoding High Dimensional Local Features by Sparse Coding Based Fisher Vectors," Nov. 2014.

[27] M. Yang, L. Zhang, X. Feng, and D. Zhang, "Sparse Representation Based Fisher Discrimination Dictionary Learning for Image Classification," Int. J. Comput. Vis., vol. 109, no. 3, pp. 209-232, 2014

[28] G. Ciuti, L. Ricotti, A. Menciassi, and P. Dario, "MEMS Sensor Technologies for Human Centred Applications in Healthcare, Physical Activities, Safety and Environmental Sensing: A Review on Research Activities in Italy," Sensors, vol. 15 , no. 3, p. 6441, 2015.

[29] Debraj De, Pratool Bharti, Sajal K. Das, Sriram Chellappan, "Multimodal Wearable Sensing for Fine-Grained Activity Recognition in Healthcare", IEEE Internet Computing, vol.19, no. 5, pp. 26-35, Sept.-Oct. 2015, doi:10.1109/MIC.2015.72

[30] C. Torres-Huitzil and A. Alvarez-Landero, "Mobile Health: A Technology Road Map," S. Adibi, Ed. Cham: Springer International Publishing, 2015, pp. 147-169.

[31] U. Fareed, "Smartphone Sensor Fusion Based Activity Recognition System for Elderly Healthcare," in Proceedings of the 2015 Workshop on Pervasive Wireless Healthcare, 2015, pp. 29-34.

[32] Ryan M. Gibson, Abbes Amira, Naeem Ramzan, Pablo Casaseca-de-la-Higuera, and Zeeshan Pervez, "Multiple Comparator Classifier Framework for Accelerometer-Based Fall Detection and Diagnostic," Applied Soft Computing, 2015.

[33] A. Avci, S. Bosch, M. Marin-Perianu, R. Marin-Perianu, and P. Havinga, "Activity Recognition Using Inertial Sensing for Healthcare, Wellbeing and Sports Applications: A Survey," in Architecture of Computing Systems (ARCS), 2010 23rd International Conference on, 2010, pp. 1-10.

[34] G. Fortino, X. Li, X. Lin, O. Mayora, E. Natalizio, and M. R. Yuce, "Wireless Technology for Pervasive Healthcare," Mob. Networks Appl., vol. 19, no. 3, pp. 273-275, 2014

[35] N. D. Lane, M. Lin, M. Mohammod, X. Yang, H. Lu, G. Cardone, S. Ali, A. Doryab, E. Berke, A. T. Campbell, and T. Choudhury, "BeWell: Sensing Sleep, Physical Activities and Social Interactions to Promote Wellbeing," Mob. Networks Appl., vol. 19, no. 3, pp. 345-359, 2014.

[36] R. I. Ramos-Garcia and A. W. Hoover, "A Study of Temporal Action Sequencing During Consumption of a Meal," in Proceedings of the International Conference on Bioinformatics, Computational Biology and Biomedical Informatics, 2013, pp. 68:68-68:75.

[37] A. Parate, M.-C. Chiu, C. Chadowitz, D. Ganesan, and E. Kalogerakis, "RisQ: Recognizing Smoking Gestures with Inertial Sensors on a Wristband," in Proceedings of the 12th Annual International Conference on Mobile Systems, Applications, and Services, 2014, pp. 149-161.

[38] Guiry, J.J.; van de Ven, P.; Nelson, J. Multi-Sensor Fusion for Enhanced Contextual Awareness of Everyday Activities with Ubiquitous Devices. Sensors 2014, 14, 5687-5701. 
International Journal of Computer Applications Technology and Research

Volume 5- Issue 9, 561 - 569, 2016, ISSN:- 2319-8656

[39] I. Sabek, M. Youssef, and A. Vasilakos, "Ace: An accurate and efficient multi-entity device-free wlan localization system," IEEE Trans. Mobile Comput., vol. 14, no. 2, pp. 261-273, Feb. 2015.

[40] Z. Sheng et al., "A survey on the ietf protocol suite for the Internet of Things: Standards,.challenges, and opportunities," IEEE Wireless Common., vol. 20, no. 6, pp. 91-98, Dec. 2013.
[41] Ö Yürür, C. H. Liu and W. Moreno, "Light-Weight Online Unsupervised Posture Detection by Smartphone Acc elerometer," in IEEE Internet of Things Journal, vol. 2, no. 4, pp. 329-339, Aug. 2015. 